\title{
Nystatin-Neomycin-Polymyxin B Combination: Efficacy and Tolerance as 1st- Line Local Treatment of Infectious Vaginitis
}

\author{
Jean-Marc Bohbot ${ }^{1}$, Patrice Sednaoui', François Verriere ${ }^{2 *}$ \\ ${ }^{1}$ Institut Alfred Fournier, Paris, France \\ ${ }^{2}$ Laboratoire Innotech International, Arcueil, France \\ Email: francois.verrriere@innothera.com
}

Received 7 April 2014; revised 5 May 2014; accepted 13 May 2014

Copyright (C) 2014 by authors and Scientific Research Publishing Inc.

This work is licensed under the Creative Commons Attribution International License (CC BY). http://creativecommons.org/licenses/by/4.0/

c) (i) Open Access

\begin{abstract}
Objective: To evaluate the efficacy and tolerance of a local treatment combining two antibacterials and one antifungal in patients with a clinical presentation suggesting infectious vaginitis. Patients and methods: 169 patients presenting with clinical criteria for vaginitis were included in an open, multicenter trial. Vaginal samples were taken for microbiological analyses and a triple-combination product of nystatin, neomycin and polymyxin $B$ was then started as local treatment, without waiting for the test results. The treatment was continued with the usual dosage (1 vaginal capsule at bedtime for 12 days) for vaginal infections in the scope of the combination product with approved labeling. A second vaginal sample was performed at the end of the treatment. The main efficacy criterion was the clinical success rate (cure or improvement of the clinical signs and symptoms) according to the investigator. Results: 93 patients were included in the efficacy population. Non-exclusively fungal vaginitis (strictly bacterial or bacterial + fungal) represented $31.2 \%$ of the cases. The clinical success rate was $97.8 \%$ according to the investigator and $95.7 \%$ according to the patients. The microbiological success rate was $81.3 \%$, with no differences between etiologies (Candida spp., bacteria or both). The combination product was well-tolerated, despite the local inflammation before treatment. Discussion and conclusion: Given the etiological diversity of vaginitis, this trial supports the efficacy of a triple-combination product (nystatin, neomycin, polymyxin B) as a first-line local treatment of Candida, bacterial or mixed vaginitis.
\end{abstract}

\section{Keywords}

Candidiasis, Mixed Vaginitis, Bacterial Vaginitis, Nystatin Neomycin, Polymyxin B, Empirical Therapy

\footnotetext{
"Corresponding author.

How to cite this paper: Bohbot, J.-M., et al. (2014) Nystatin-Neomycin-Polymyxin B Combination: Efficacy and Tolerance as 1st- Line Local Treatment of Infectious Vaginitis. Open Journal of Obstetrics and Gynecology, 4, 445-454.

http://dx.doi.org/10.4236/ojog.2014.47065
} 


\section{Introduction}

Vaginitis represents a very common reason for consultation in gynecology [1]. It is worth considering that the most common causes are infectious: up to $90 \%$ of consultations for vaginitis are related to a vulvovaginal candidiasis (VVC), bacterial, bacterial vaginosis or trichomoniasis [2].

Infectious vaginitis is characterized by the appearance of unusual vaginal discharge associated with inflammatory symptoms (itching, painful, vaginal erythema and dyspareunia) [3]. Vaginitis differs from bacterial vaginosis through the presence of local inflammation [4].

The clinical presentation and the speculum examination can sometimes suggest a particular etiology (Candida, Trichomonas). However, the diagnosis is more difficult to establish in the case of a mixed or bacterial etiology [5]. Indeed, in $15 \%$ - $40 \%$ of vulvovaginal candidiasis, bacterial superinfection is found but generally the clinical manifestations of mycosis predominate [6]-[11]. In addition, although most cases of vulvovaginal mycosis are due to Candida albicans, there is an emergence of non-albicans Candida fungal infections (e.g., Candida glabrata) representing up to $15 \%$ of patients [12] [13].

The microbiological analysis of vaginal swabs therefore seems to be the test of choice that makes it possible to identify the pathogen (s) responsible for the infection. However, for economic reasons, due to a lack of adequate facilities or for medical reasons, it is not always easy to prescribe a vaginal microbiological examination to all women with vaginitis. Thus, in nearly half of vaginitis consultations, no vaginal swab is carried out [14]. Moreover, it is now established that the microbiological analysis, reliable in confirming the presence of inflammation and rather reliable in confirming the presence of yeast, becomes unreliable in the case of bacterial superinfection and is not at all effective in identifying bacteria implicated, if there are any [3] [15]. Bacteriology can give false negative results in almost $40 \%$ of cases, the results being highly variable according to the sampling site, the time of a day at which it is carried out and the phase of the patient's menstrual cycle. False positives may also be obtained in the case of asymptomatic carriers of bacterial germs [5] [16] [17]. Finally, the results of microbiological analysis are obtained after a few days whereas vaginitis requires immediate care. Therefore, an empirical treatment is often initiated as the 1st-line to quickly relieve the patient [5]. In the case of typical vulvovaginal mycosis, for example, an empirical treatment using vaginal or sometimes oral antifungals (azoles) is generally used [18].

This study evaluates the efficacy and tolerance of the combination of two antibacterial agents (neomycin, polymyxin B) and an antifungal (nystatin) as 1st-line local treatment of microbiologically confirmed infectious vaginitis. The combination of these three active ingredients within a vaginal capsule has been marketed in France since 1969 (NNP combination; Polygynax ${ }^{\circledR}$, Innotech International Laboratory). Thanks to its broad spectrum of action, the NNP combination covers the majority of pathogens frequently involved in fungal and/or bacterial vaginitis [19]-[24]. However, despite its widespread use, recent clinical data on the safety and efficacy of this product are required to confirm the suitability of the product to the current epidemiology of vaginitis in France [6].

The objectives of this multicenter study were firstly to update the clinical efficacy, microbiological, and tolerance data for the NNP combination and secondly to compare the clinical and microbiological normalization of patients after treatment.

\section{Patients and Methods}

\subsection{Study Design}

This was an open, prospective and multicenter study with centralized microbiology, which ran from April to November 2009 in gynecology consultation in metropolitan France. The protocol (Eu-draCT No. 2008-00787439) was performed according to the Declaration of Helsinki and French law for biomedical research and received approval from the French Ethics Committee (Île-de-France VII Comité de protection des personnes, Paris).

\subsection{Patients}

The patients included in this study were aged between 18 and 65 years old and presented with clinical symptoms of mycosis and/or bacterial vaginitis (unusual vaginal discharge, itching, painful urination, dyspareunia). The 
main exclusion criteria were as follows: sexually transmitted infections (STIs), pregnancy, bacterial vaginosis and concomitant administration of local or systemic anti-fungal agents. Written consent was obtained after informing each patient.

\subsection{Treatment}

All patients received the study treatment from the outset, without waiting for the results of the microbiological examination of their vaginal swab. Following the results of this examination, the treatment was either continued at the usual dosage (vaginitis as indicated by the Marketing Authorization) or interrupted (normal microbiological examination, bacterial vaginosis, STI or germs for which a specific treatment is required). Treatment consisted of the administration of a soft vaginal capsule daily at bedtime for 12 days, containing a combination of two bactericidal antibiotics (neomycin and polymyxin B, 35,000 IU each) and an antifungal (nystatin, 100,000 IU). The neomycin-nystatin-polymyxin B combination (NNP combination) is marketed under the name Polygynax $^{\circledR}$ (Laboratoire Innotech International, Arcueil, France). Neomycin is a broad spectrum aminoglycoside antibiotic, active against Gram-negative and some Gram-positive bacteria. Polymyxin B is a polypeptidic antibiotic with antimicrobial activity on Gram-negative bacteria. Nystatin is a polyene antifungal agent used as local treatment of yeast infections (Candida albicans and non-albicans).

Patient compliance with treatment was determined by the number of unused vaginal capsules, which were collected at the final visit. Compliance is expressed as a percentage, according to the following calculation: [(number of capsules given - number of reported capsules)/(date of last dose - date of 1 st dose +1$)] \times 100$. This calculation takes into account the number of capsules and the duration of treatment.

\subsection{Clinical Evaluation}

The efficacy criteria were based on the clinical success after treatment according to the investigator.

The investigator's evaluation was carried out following a gynecological speculum examination of the cervix and vaginal wall, and questioning of the patient. How the patients felt was also collected during the study, with an intensity score associated with each clinical symptom of vaginitis.

\subsection{Microbiological Evaluation}

For each patient, samples of vaginal secretions were carried out by the investigator for microbiological analysis, firstly the inclusion visit, and secondly at the final visit at the end of treatment. These samples were taken using sterile swabs at the lateral or anterior vaginal cul-de-sac and sent to a centralized microbiology laboratory in semisolid transport medium (Alfred Fournier Institute Laboratory, Paris). Microbiological analyses, performed according to the protocol described by Bohbot et al., 2011, were interpreted in blinded fashion [6]. Microbiological results were obtained between 3 and 5 days. In this study, only yeast or bacteria present in the direct microscopic examination and isolated in significant amounts in culture were considered pathogenic.

\subsection{Evaluation of Safety}

The safety of the NNP combination was evaluated based on adverse events (AEs) reported during the study for all patients who received at least one dose of study treatment, including those which were not eligible for the evaluation of treatment efficacy (normal microbiological examination, bacterial vaginosis, STI or germs for which a specific treatment is required). The evaluation of tolerance therefore focused on all patients included in the study $(\mathrm{n}=169)$.

\subsection{Evaluation Criteria and Statistical Analysis}

The primary endpoint was the clinical efficacy evaluated by the proportion of patients with clinical success at the final visit. Clinical success was defined as the cure or improvement of functional symptoms of vaginitis according to the investigator's evaluation. The intent-to-treat (ITT) population included patients who received at least one dose of study treatment and were evaluated for the effectiveness of treatment after 15-17 days (final visit). A per-protocol analysis (PP) was also performed, including patients in the ITT population with no major protocol deviation $(\mathrm{n}=87)$. 
Other criteria studied were the microbiological success rate, the clinical success rate according to the patient and the evolution of symptoms (unusual vaginal discharge, pruritus, dyspareunia, painful urination) by scoring and adding up the intensity of each symptom (absent $=0$, moderate $=1$, severe $=2$ ). Microbiological treatment success was defined as the disappearance or improvement of the microbiological anomaly observed in the analysis, without any new pathogen (bacterial or yeast).

The agreement between the clinical and microbiological success rates was evaluated by calculating the Kappa coefficient corresponding to the ratio of the proportion of agreement observed due to chance over the potential agreement [25].

Finally, the quantitative data are presented as the mean with the standard deviation. The qualitative data are expressed as the number and percentage of patients. The mean sum of symptoms intensity scores was compared before and after treatment with a Student's t test on paired series. The proportions of patients with positive microbiological analyses at the beginning and the end of treatment were compared using McNemar's test. Statistical analyzes were performed using the SAS 9.1 software (SAS Institute Inc., Cary, NC, USA).

\section{Results}

\subsection{Patient Characteristics}

A total of 169 patients were included based on clinical criteria of vaginitis following gynecological examination and questioning. All patients received at least one capsule of NNP combination as 1st-line treatment, in other words, without waiting for the results of the microbiological analysis. Upon receipt of these results, 69 patients were excluded from the study for the following reasons: strictly normal microbiological examination $(n=51)$, specific germs $(n=16)$, non-exploitable sample $(n=2)$. Of the 100 patients eligible for the evaluation of NNP combination effectiveness, 93 were included in the intent-to-treat population (ITT, $n=93$ ). Within this population, 6 patients presented major deviations from the protocol and were excluded from the per-protocol population (PP, $\mathrm{n}=87$ ). The disposition of patients in the study is detailed in Figure 1.

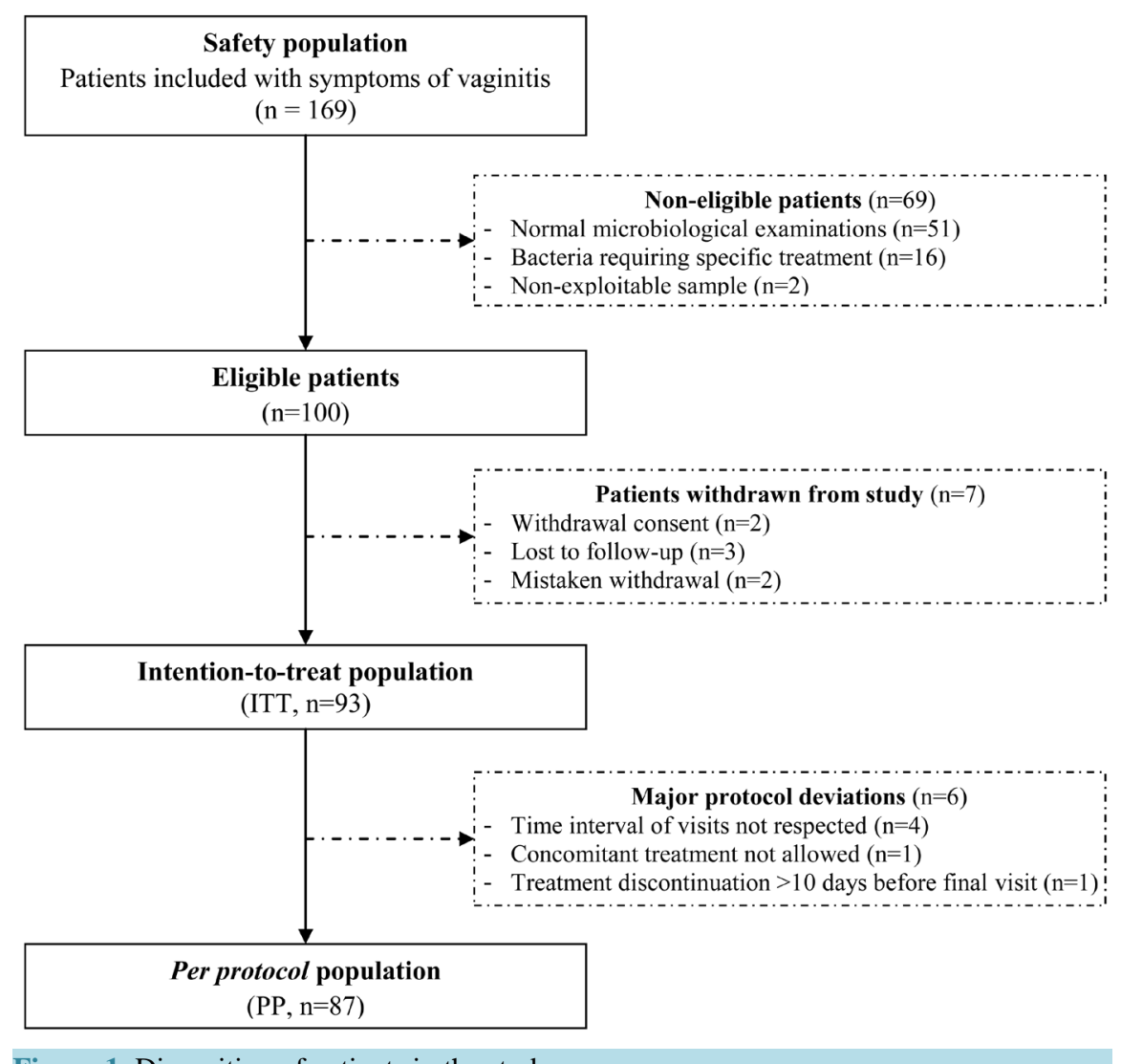

Figure 1. Disposition of patients in the study. 
The clinical and demographic patient characteristics of the ITT population are described in Table 1. The mean age of the patients was $33.1 \pm 11.7$ years.

Among the functional symptoms of vaginitis, unusual vaginal discharge and pruritus were the most common and were found in $94.7 \%$ and $87.1 \%$ of patients, respectively. Painful urination was found in $45.2 \%$ of patients and dyspareunia was found in $63 \%$ of sexually active patients $(n=68)$. The duration of the symptoms of vaginitis was an average of $17.5 \pm 33.8$ days.

The results of the microbiological analyses at baseline are detailed in Table 2. In the ITT population, the most common etiology was candidiasis, with $68.8 \%$ (64/93) of cases. The vast majority of these vaginitis were due to the yeast Candida albicans, but non-albicans Candida were isolated in $3.1 \%$ of cases of (2/64). Non-exclusively fungal vaginitis (strictly bacterial or bacterial + fungal) accounted for 31.2\% (29/93) of patients, of which 62\% $(18 / 29)$ had a polymorphic bacterial flora, in other words, combining several pathogens but without a predominant one.

The kappa coefficient of agreement between the clinical diagnosis established by the investigator and the microbiological diagnosis of infectious vaginitis was $0.07(95 \% \mathrm{CI}=[-0.33 ; 0.47])$ in the ITT population.

\subsection{Evaluation of the Effectiveness of Treatment}

In the ITT population and according to the investigators, clinical success was observed in 91 of 93 patients (70 patients cured and 21 patients improved), a clinical success rate of $97.8 \%$. Depending on the etiology of vaginitis, the rate ranged from $95.8 \%$ for candidiasis to $100 \%$ for mixed vaginitis combining bacterial and fungal infection (Table 3). According to the patients, 89 of 93 felt they had achieved clinical success, which is a rate of 95.7\%. The presence and severity of clinical symptoms associated with vaginitis were improved between the first and the last visit after treatment. Indeed, the frequencies of unusual vaginal discharge, pruritus, dyspareunia and painful urination decreased by $72.7 \%$ (from 88 to 24 patients), $90.1 \%$ (from 81 to 8 patients), $76.7 \%$ (43 to 10 patients) and $85.7 \%$ (from 42 to 6 patients). The intensity of symptoms, estimated by the mean sum of each symptom intensity scores, decreased statistically significantly $(\mathrm{p}<0.0001)$ between baseline $(3.85 \pm 1.33)$ and the end of the study $(0.57 \pm 0.95)$.

As regards the analysis of the vaginal swabs, the proportion of patients with microbiological anomaly decreased statistically after treatment (from 74 to 17 infected patients, $\mathrm{p}<0.0001$ ). Thus, microbiological success, evaluated in 91 patients in the ITT population (data not available for 2 patients) was found for 74 of them representing a rate of $81.3 \%$. Agreement between the investigator's evaluation and the microbiological analysis

Table 1. Patient characteristics at inclusion (Intention-to-treat (ITT) population).

\begin{tabular}{|c|c|}
\hline Patient characteristics & \\
\hline Sample size, number & $\mathrm{n}=93$ \\
\hline Age in years (mean \pm standard deviation) & $33.1 \pm 11.7$ \\
\hline Body mass index, $\mathrm{kg} / \mathrm{m}^{2}$ (mean \pm standard deviation) & $21.9 \pm 3.4$ \\
\hline \multicolumn{2}{|l|}{ Clinical symptoms of vaginitis } \\
\hline Unusual vaginal discharge, n (\%) & 88 (94.7) \\
\hline Moderate & $58(62.4)$ \\
\hline Severe & 30 (32.3) \\
\hline Pruritus, n (\%) & $81(87.1)$ \\
\hline Moderate & 35 (37.6) \\
\hline Severe & $46(49.5)$ \\
\hline Dyspareunia, n (\%) & $43(46.3)$ \\
\hline Moderate & $25(26.9)$ \\
\hline Severe & $18(19.4)$ \\
\hline Non-applicable & 25 (26.9) \\
\hline Painful urination, n (\%) & $42(45.2)$ \\
\hline Moderate & $36(38.7)$ \\
\hline Severe & $6(6.5)$ \\
\hline \multicolumn{2}{|l|}{ Score and duration of symptoms } \\
\hline Overall score of severity of symptoms & $3.85 \pm 1.33$ \\
\hline Duration of symptoms before inclusion, days (mean \pm standard deviation) & $17.5 \pm 33.8$ \\
\hline
\end{tabular}


Table 2. Microbiological test results at baseline (ITT population, $\mathrm{n}=93$ ).

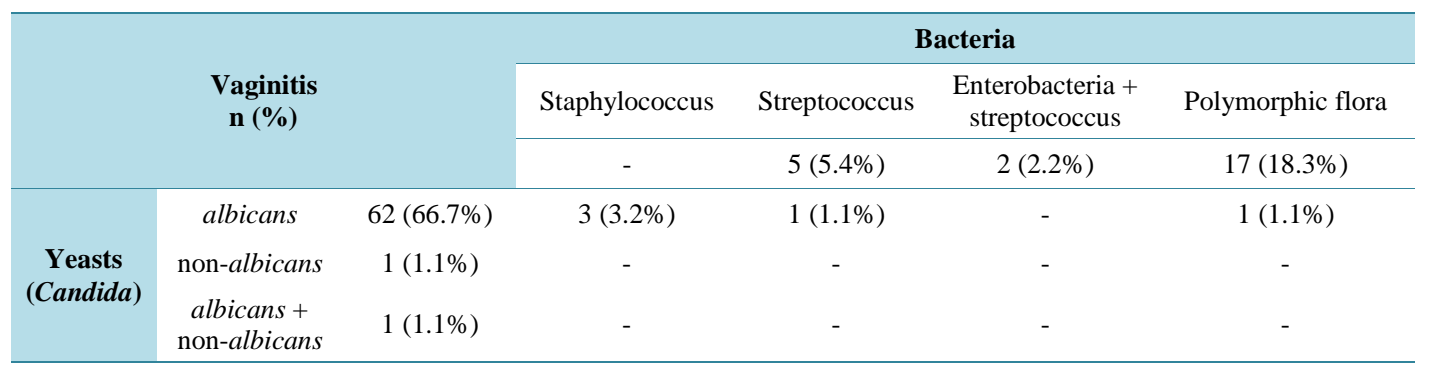

Table 3. Clinical and microbiological efficacy of the NNP combination as 1st-line local treatment in patients with a microbiologically confirmed vaginitis (ITT population).

\begin{tabular}{cc|}
\hline Investigators' opinion (primary endpoint) & \\
\hline Clinical success rate, n (\%) & $91 / 93(97.8)^{*}$ \\
Candida vaginitis & $63 / 64(98.4)$ \\
Bacterial vaginitis & $23 / 24(95.8)$ \\
Mixed vaginitis (Candida and bacterial) & $5 / 5(100)$ \\
\hline Patients' opinion (secondary endpoint) & \\
\hline Clinical success rate, n (\%) & $89 / 93(95.7)$ \\
\hline Cure/Improvement of clinical symptoms of vaginitis & $64 / 88(72.7)$ \\
Unusual vaginal discharge, n (\%) & $73 / 81(90.1)$ \\
Pruritus, $n$ (\%) & $33 / 43(76.7)$ \\
Dyspareunia, n (\%) & $36 / 42(85.7)$ \\
Painful urination, $n$ (\%) & $74 / 91(81.3)$ \\
\hline
\end{tabular}

*70 patients cured and 21 improved.

Table 4. Clinical and microbiological efficacy of the NNP combination: agreement between the opinion of the investigator and the microbiological analysis after treatment (ITT population).

\begin{tabular}{cccc}
\hline \multirow{2}{*}{$\begin{array}{c}\text { Agreement between the opinion of the investigator } \\
\text { and the microbiological analysis, } n(\%)\end{array}$} & \multicolumn{2}{c}{ Investigator's opinion } \\
\cline { 2 - 4 } & Success $^{2} \mathrm{n}=74$ & $73(78.5)$ & Failure $\mathrm{n}=2$ \\
\hline \multirow{2}{*}{ Microbiological analysis $\mathrm{n}=91$} & Failure $\mathrm{n}=17$ & $16(17.2)$ & $1(1.1)$ \\
& Missing data $\mathrm{n}=2$ & $2(2.9)$ & $-1.1)$ \\
\hline
\end{tabular}

${ }^{1}$ According to the investigator's opinion, clinical success is defined as the cure or improvement of the functional signs of vaginitis.

${ }^{2}$ The microbiological success of the treatment is defined as the disappearance or improvement of the anomaly.

was found for 74 patients, or $81.3 \%$ fit (Table 4). Moreover, for 2 patients deemed to have failed treatment by the investigator, the initial microbiological anomaly had disappeared after treatment.

The results of the per-protocol analysis $(n=87)$ confirmed those obtained for the ITT population $(n=93)$. The proportion of patients with clinical success was $97.7 \%$ (85/87) according to the investigators, $95.4 \%$ (83/87) according to the patients' opinion and $82.4 \%$ (70/87) according to the microbiological analysis of vaginal swabs (results not shown).

\subsection{Compliance and Treatment Tolerance}

In the ITT population, compliance to the NNP combination was evaluated in 90 patients who brought back their treatment boxes. The mean compliance of these patients was $99.6+/-2.2 \%$. In theory, the duration of treatment 
for NNP combination is 12 days. The mean duration of treatment in this study was $11.7+/-1.3$ days.

The tolerance for the NNP combination was evaluated for the total number of patients included $(\mathrm{n}=169)$ because they all received at least one vaginal capsule while waiting for the results of the vaginal swabs. No serious adverse event (AE) was reported during this trial. Five adverse events were reported by 5 patients (3\%): herpes on the neck, tonsillitis, uterine bleeding, urinary burning and vaginal candidiasis. The treatment as the cause of urinary burning and mycosis was deemed "possible” and "probable” by the investigators, respectively, leading to discontinuation of treatment for these 2 patients (1.2\%). However, the development of vaginal yeast infection was found in one patient whose microbiological examination was initially normal and who received the NNP combination from the previous day. In addition, the diagnosis of vaginal mycosis was established on the basis of clinical examination only and no microbiological test was carried out to confirm it. Painful urination was reported by one patient with Candida vaginitis after 3 days of treatment. This AE was resolved without corrective treatment and the microbiological analysis carried out on the final visit indicated complete disappearance of the initial anomaly.

The other 3 AEs were not attributed to the treatment. All events resolved without sequelae.

\section{Discussion}

The etiological diversity of vaginitis is well established. It was found in the distribution of pathogens incriminated in this study that included 169 patients on the basis of a clinical presentation suggestive of infectious vaginitis [6]. After microbiological analysis of vaginal samples, the infectious etiology of vaginitis represented nearly $70 \%$ of cases.

According to studies, this proportion is highly variable [2] [8] [11], probably due to geographical disparity and the size of the cohorts studied.

Non-exclusively fungal vaginitis (bacterial infections that are pure or combined with a mycosis) represented $31.2 \%(29 / 93)$ of the ITT population. These results are lower than those obtained in recent studies that reported more frequent mixed and bacterial vaginitis: $56.7 \%$ of 434 patients and $79.2 \%$ of 447 patients in Turkey [10] [11]; 71.6\% of 225 patients in the Czech Republic [8]; 87.2\% of 172 patients in India [9]. These discrepancies can be explained in part by the epidemiology of each country and also by the type of vaginitis included in the studies.

In any event, the significant prevalence of non-exclusively fungal infections suggests that 1st-line antifungal treatment as monotherapy may be ineffective in a significant number of cases. A broad spectrum empirical treatment combining antifungal and antibacterial activities appears to be the best option for a complete clinical cure.

In this study, non-albicans Candida were isolated in 3.1\% (2/64) of cases of mycotic vaginitis. Similarly, this relatively low prevalence of non-albicans Candida in vaginal fungal infections is lower than that reported in the literature. Indeed, an American study showed an incidence of 10\% - 80\% of non-albicans Candida in fungal vaginitis following the reporting countries [26].

The presence of non-albicans Candida is an important consideration because the azole treatments commonly used in 1st-line treatment (e.g., econazole or fluconazole) show inconsistent activity on non-albicans Candida [27] [28]. This is not the case for nystatin, one of the three components of the NNP combination tested in this study, which remains effective on both albicans and non-albicans Candida species [28]-[30].

Microbiological analyses of vaginal samples before treatment were compared to the clinical diagnosis made by the investigators at baseline, on the basis of clinical examination and questioning of the patient. Some clinical symptoms are indeed suggestive of a specific etiology, such as lumpy vaginal discharge associated with intense pruritus, suggesting candidiasis or foamy and greenish vaginal discharge, suggesting a Trichomonas infection. However, the diagnosis is more difficult to establish in the case of mixed etiology. Indeed, several studies have shown that the predictive value of clinical symptoms is low [5] [16] [17] [31]. In a study comparing clinical and biological diagnoses of 220 vaginitis, Schwiertz et al. [32] found 77\% misdiagnosis in the case of candidiasis and $87 \%$ in the case of mixed vaginitis. The present study confirms the difficulty of establishing an accurate diagnosis on the basis of the clinical presentation alone. In the first place, in $30 \%$ of cases of vaginitis included in this study, an infectious etiology had been suggested, erroneously. In addition, in infectious vaginitis, this study did not reveal any correlation between the clinical and the microbiological diagnosis (kappa $=0.07)$. These results confirm the data in the literature suggesting that the clinical examination does not exclude the possibility of 
a mixed infection. Also, the use in empirical treatment of a broad spectrum anti-fungal and anti-bacterial combination seems to be the most appropriate therapeutic approach for vaginitis.

The clinical efficacy of the NNP combination was clearly demonstrated with a success rate according to the investigators of $97.8 \%$ in 93 patients with microbiologically confirmed infectious vaginitis. This result was confirmed by the overall evaluation by the patients (clinical success rate: 95.7\%) and their feelings about each symptom (pruritus, painful urination, unusual vaginal discharge, dyspareunia). These results complement those of a previous efficacy study carried out on the NNP combination in 121 patients with a non-specific infectious vaginitis which showed a clinical success rate of $92.2 \%$ according to the patients [22].

In this study, the clinical efficacy of the NNP combination seemed independent of the infectious etiology as it was demonstrated for candidiasis (98.4\%), for candidiasis with bacterial superinfection (100\%) and for bacterial vaginitis (95.8\%).

In addition, the retained effectiveness against non-albicans Candida infections $(100 \%, 2 / 2)$ is important because of the emergence of this type of infection and increased resistance to azoles [12] [13] [27].

The clinical efficacy according to the opinion of the investigators was supplemented by the microbiological analysis of vaginal swabs after treatment $(\mathrm{n}=91)$. This analysis indicated complete disappearance of the identified pathogen in 74 patients, which represents a microbiological success rate of $81.3 \%$. These results are consistent with those of Bruhat et al. study, who obtained a cumulative rate of clinical success according to the investigator and microbiological success of $76.6 \%$ after 12 days of treatment [22].

Agreement with the opinion of the investigators was also noted for 74 patients (73 successes, 1 failure). Among patients who were considered as "microbiological failure", it is worth distinguishing those with an improvement in flora without complete disappearance of the pathogen (4/91) from those with a persistently disturbed flora (13/91). Furthermore, among these last 13 patients, 12 were cured according to the investigator and/or the patient herself. This result highlights the limitations of microbiological analysis. Polymorphism of the vaginal microbiota and its complexity can sometimes generate false interpretations of the microbiological analysis, particularly among asymptomatic carriers of pathogens and when comparing results before and after treatment [3] [15]. However, in this case, it is possible that the period between the end of the study and the microbiological test was too short to allow for complete rebalancing of the vaginal flora. Therefore, to be sure of a cure or improvement of infectious vaginitis, three items must be taken into account: the clinical examination, the opinion of the patient and the improvement of the symptoms. The medical opinion and that of the patient seem more relevant to evaluate the effectiveness of treatment, because they better reflect reality than the microbiological analysis. Clinical success is achieved when the patient is relieved and the clinical examination is normal, which was observed in over 95\% of cases in this study. The limitations of microbiological analysis highlighted in this study and the convergence of opinions between investigators and patients justify carrying out such an open study. Indeed, open study is more akin to general practice for which, in the absence of microbiological analysis, a 1st-line empirical treatment is often used.

Despite local inflammation associated with vaginitis, tolerance of the NNP combination was very good. Five adverse events were reported in this study, which is $3 \%$ of $\mathrm{AE}$ and only two were considered possibly or probably due to the treatment. This rate of $\mathrm{AE}$ is lower than those reported in the Cochrane review: from $12 \%$ for econazole and miconazole to $21 \%$ and $22 \%$ for fluconazole and clotrimazole, respectively [33].

The relatively short follow-up of patients meant it was not possible to estimate the rate of relapse and/or recurrence after treatment. However, recent data obtained in vitro indicate that three main strains of vaginal lactobacilli are resistant to the NNP combination [34]. With the vast majority of lower urogenital infections in women due to an imbalance of this protective flora, its balancing-up with the NNP combination could be a strong argument in favor of limiting the recurrence after treatment. Further studies are needed to confirm these data.

\section{Conclusion}

Given the etiological diversity of infectious vaginitis, this study confirms that the NNP combination (nystatin, neomycin, polymyxin B) is appropriate as a 1st-line local treatment of this common condition. Its broad spectrum of action allows a high clinical efficacy on the most frequent germs encountered in vaginitis. Furthermore, the retained efficiency against Candida spp. is an asset, especially when faced with variable sensitivity to azoles of non-albicans strains. In clinical practice, the NNP combination is suitable for the empirical treatment of vaginitis, in the absence of or pending microbiological results. 


\section{Conflicts of Interests}

This study was promoted and funded by Innotech International Laboratory. Dr. J.-M. Bobhot: principal investigator and study coordinator, Medical Director of Alfred Fournier Institute. Dr. P. Sednaoui: co-investigator of the study, Microbiology Laboratory in the Alfred Fournier Institute. Dr. F. Verrière: Medical Director, Innotech International Laboratory.

\section{References}

[1] ACOG Practice Bulletin (2006) Clinical Management Guidelines for Obstetrician—Gynecologists. American Journal of Obstetrics and Gynecology, 107, 1195-1206.

[2] Egan, M.E. and Lipsky, M.S. (2000) Diagnosis of Vaginitis. American Family Physician, 62, 1095-1104.

[3] Sobel, J.D. (1997) Vaginitis. The New England Journal of Medicine, 337, 1896-1903. http://dx.doi.org/10.1056/NEJM199712253372607

[4] Donders, G.G., Vereecken, A., Bosmans, E., Dekeersmaecker, A., Salembier, G. and Spitz, B. (2002) Definition of a Type of Abnormal Vaginal Flora That Is Distinct from Bacterial Vaginosis: Aerobic Vaginitis. BJOG: An International Journal of Obstetrics \& Gynaecology, 109, 34-43. http://dx.doi.org/10.1111/j.1471-0528.2002.00432.x

[5] Landers, D.V., Wiesenfeld, H.C., Heine, R.P., Krohn, M.A. and Hillier, S.L. (2004) Predictive Value of the Clinical Diagnosis of Lower Genital Tract Infection in Women. American Journal of Obstetrics \& Gynecology, 190, 1004-1008. http://dx.doi.org/10.1016/j.ajog.2004.02.015

[6] Bohbot, J.M., Sednaoui, P., Verriere, F. and Achhammer, I. (2012) The Etiologic Diversity of Vaginitis. Gynécologie Obstétrique \& Fertilité, 40, 578-581. http://dx.doi.org/10.1016/j.gyobfe.2011.08.001

[7] Eckert, L.O. (2006) Clinical Practice. Acute Vulvovaginitis. The New England Journal of Medicine, 355, 1244-1252. http://dx.doi.org/10.1056/NEJMcp053720

[8] Libalova, Z., Cepicky, P., Malina, J., Stanslicky, K., Kuzelova, M., Medalova, Z., et al. (2007) Vulvovaginitis. Occurrence and Importance of Mixed and Unclassifiable Pictures. Ceská Gynekologie, 72, 32-37.

[9] Thulkar, J., Kriplani, A., Agarwal, N. and Vishnubhatla, S. (2010) Aetiology \& Risk Factors of Recurrent Vaginitis \& Its Association with Various Contraceptive Methods. Indian Journal of Medical Research, 131, 83-87.

[10] Kalkanci, A., Ciftçi, B., Biri, A., Kustimur, S. and Güner, H. (2005) Distribution of the Pathogens According to Vaginal Culture Results of the Patients with Vaginitis. Türkiye Klinikleri Jinekoloji Obstetrik Özel Dergisi, 15, 137-139.

[11] Ozturk, C.E., Ozdemir, I., Yavuz, T., Kaya, D. and Behcet, M. (2006) Etiologic Agents of Cervicovaginitis in Turkish Women. Saudi Medical Journal, 27, 1503-1507.

[12] Fidel Jr., P.L., Vazquez, J.A. and Sobel, J.D. (1999) Candida Glabrata: Review of Epidemiology, Pathogenesis, and Clinical Disease with Comparison to C. albicans. Clinical Microbiology Reviews, 12, 80-96.

[13] Richter, S.S., Galask, R.P., Messer, S.A., Hollis, R.J., Diekema, D.J. and Pfaller, M.A. (2005) Antifungal Susceptibilities of Candida Species Causing Vulvovaginitis and Epidemiology of Recurrent Cases. Journal of Clinical Microbiology, 43, 2155-2162. http://dx.doi.org/10.1128/JCM.43.5.2155-2162.2005

[14] Wiesenfeld, H.C., et al. (1999) The Infrequent Use of Office-Based Diagnostic Tests for Vaginitis. American Journal of Obstetrics \& Gynecology, 181, 39-41. http://dx.doi.org/10.1016/S0002-9378(99)70433-3

[15] Larsen, B. (1993) Vaginal Flora in Health and Disease. Clinical Obstetrics \& Gynecology, 36, 107-121. http://dx.doi.org/10.1097/00003081-199303000-00016

[16] Schaaf, V.M., Perez-Stable, E.J. and Borchardt, K. (1990) The Limited Value of Symptoms and Signs in the Diagnosis of Vaginal Infections. Archives of Internal Medicine, 150, 1929-1933. http://dx.doi.org/10.1001/archinte.1990.00390200111021

[17] Lurie, S., Woliovitch, I., Rotmensch, S., Sadan, O. and Glezerman, M. (2001) Value of Vaginal Culture in Management of Acute Vaginitis. Archives of Gynecology and Obstetrics, 265, 187-189. http://dx.doi.org/10.1007/s004040000157

[18] González, I.D.C., González, F.G., Cuesta, T.S., Fernández, J.M., Rodríguez, J.M.D.A., Ferrairo, R.A.E., et al. (2011) Patient Preferences and Treatment Safety for Uncomplicated Vulvovaginal Candidiasis in Primary Health Care. BMC Public Health, 11, 63. http://dx.doi.org/10.1186/1471-2458-11-63

[19] Jacquetin, B. and Wattiez, A. (1986) Intérêt de l'utilisation de Polygynax sans acétarsol dans le traitement de l'infection génitale basse. Revue de Médecine de Clermont-Ferrand, 15, 32-36.

[20] Marhic, C. and Charles, G. (1986) Traitement local des vaginites microbiennes par une association nystatine, néomycine, polymyxine B. Gazette médicale de France, 93, 83-84. 
[21] Blanc, B. (1987) Le traitement des infections cervico-vaginales par Polygynax sans acetarsol. Méditerranée Médicale, 15, 33-35.

[22] Bruhat, M.A. (1992) Use of a Local Antibiotic Combination in the Treatment of Mycotic Vaginitis and Mixed Vaginitis. Revue Française de Gynecologie et d Obstetrique, 87, 161-168.

[23] Goran, D., Vesna, A., Adela, S., Biljana, T.K. and Snezana, M. (2004) Polygynax in the Treatment of Fungal and Non Specific Vaginitis. Akusherstvo i Ginekologiia (Sofiia), 43, 23-26.

[24] Verriere, F. (2011) Effectiveness of Polygynax in the Treatment of Vaginitis: A Prospective Multicentre Study. Bulgarian Society of Obstetrics and Gynecology, 50, 32-37.

[25] Schwartz, D. (1996) Méthodes statistiques à l'usage des médecins et biologistes. $4^{\text {ème }}$ édition. Collection statistique en biologie et en médecine, Médecine-Science Flammarion.

[26] Achkar, J.M. and Fries, B.C. (2010) Candida Infections of the Genitourinary Tract. Clinical Microbiology Reviews, 23, 253-273. http://dx.doi.org/10.1128/CMR.00076-09

[27] Danby, C.S., Boikov, D., Rautemaa-Richardson, R. and Sobel, J.D. (2012) Effect of pH on in Vitro Susceptibility of Candida glabrata and Candida albicans to 11 Antifungal Agents and Implications for Clinical Use. Antimicrobial Agents and Chemotherapy, 56, 1403-1406. http://dx.doi.org/10.1128/AAC.05025-11

[28] Fan, S.R., Liu, X.P. and Li, J.W. (2008) Clinical Characteristics of Vulvovaginal Candidiasis and Antifungal Susceptibilities of Candida Species Isolates among Patients in Southern China from 2003 to 2006. Journal of Obstetrics and Gynaecology Research, 34, 561-566. http://dx.doi.org/10.1111/j.1447-0756.2008.00817.x

[29] Fan, S.R. and Liu, X.P. (2011) In Vitro Fluconazole and Nystatin Susceptibility and Clinical Outcome in Complicated Vulvovaginal Candidosis. Mycoses, 54, 501-505. http://dx.doi.org/10.1111/j.1439-0507.2010.01887.x

[30] Choukri, F., Benderdouche, M. and Sednaoui, P. (in press) In Vitro Susceptibility Profile of 200 Recent Clinical Isolates of Candida spp. to Topical Antifungal Treatments of Vulvovaginal Candidiasis, the Azoles and Nystatin Agents. Journal of Medical Mycology.

[31] Bornstein, J., Lakovsky, Y., Lavi, I., Bar-Am, A. and Abramovici, H. (2001) The Classic Approach to Diagnosis of Vulvovaginitis: A Critical Analysis. Infectious Diseases in Obstetrics and Gynecology, 9, 105-111. http://dx.doi.org/10.1155/S1064744901000187

[32] Schwiertz, A., Taras, D., Rusch, K. and Rusch, V. (2006) Throwing the Dice for the Diagnosis of Vaginal Complaints? Annals of Clinical Microbiology and Antimicrobials, 5, 4. http://dx.doi.org/10.1186/1476-0711-5-4

[33] Nurbhai, M., Grimshaw, J., Watson, M., Bond, C., Mollison, J. and Ludbrook, A. (2007) Oral versus Intra-Vaginal Imidazole and Triazole Anti-Fungal Treatment of Uncomplicated Vulvovaginal Candidiasis (Thrush). Cochrane Database of Systematic Reviews, 17, Published Online.

[34] Neut, C., Nelis, H.J. and Coenye, T. (2012) Treatment of Infectious Vaginitis: Antimicrobial Drugs Effects on Lactobacillus strains of the Normal Vaginal Flora. Internal Study Report. 